\title{
Training for Deep Brain Stimulation Planning Using Objective Assessment
}

\author{
Matthew S. Holden ${ }^{1}$, Yulong Zhao ${ }^{2}$, Claire Haegelen ${ }^{3}$, Caroline Essert ${ }^{4}$, Sara \\ Fernandez-Vidal ${ }^{5}$, Eric Bardinet ${ }^{5}$, Tamas Ungi ${ }^{1}$, Gabor Fichtinger ${ }^{1}$, Pierre Jannin ${ }^{2}$ \\ ${ }^{1}$ Laboratory for Percutaneous Surgery, School of Computing, Queen's University, \\ Kingston, Canada \\ ${ }^{2}$ Equipe MediCIS, Faculté de Médicine, Université de Rennes 1, Rennes, France \\ ${ }^{3}$ Centre Hospitalier Universitaire de Rennes, Rennes, France \\ ${ }^{4}$ ICube, Université de Strasbourg, Strasbourg, France \\ ${ }^{5}$ ICM CENIR, Hôpital Pitié-Salpêtrière, Paris, France \\ *Email: 72mh@queensu.ca
}

\begin{abstract}
Objective: Deep brain stimulation (DBS) is an increasingly common treatment for neurodegenerative diseases. Neurosurgeons must have thorough procedural, anatomical, and functional knowledge to plan electrode trajectories and thus ensure treatment efficacy and patient safety. Developing this knowledge requires extensive training. We propose a training approach with objective assessment of neurosurgeon proficiency in DBS planning.

Methods: To assess proficiency, we propose analyzing both the viability of the planned trajectory and the manner in which the operator arrived at the trajectory. To improve understanding, we suggest a self-guided training course for DBS planning using real-time feedback. To validate the proposed measures of proficiency and training course, two experts and six novices followed the training course, and we monitored their proficiency measures throughout.

Results: At baseline, experts planned higher quality trajectories and did so more efficiently. As novices progressed through the training course, their proficiency measures increased significantly, trending toward expert measures.

Conclusion: We developed and validated measures which reliably discriminate proficiency levels. These measures are inte- grated into a training course, which quantitatively improves trainee performance. The proposed training course can be used to improve trainees' proficiency, and the quantitative measures allow trainees' progress to be monitored.
\end{abstract}

Keywords: Deep Brain Stimulation, objective skill assessment, simulation-based training 


\section{Introduction}

Deep brain stimulation (DBS) is a delicate procedure involving the placement of thin electrodes into targets in the basal ganglia. DBS is used to treat motor diseases such as Parkinson's disease or essential tremor as well as other neurological diseases including obsessive compulsive disorder and depression [1]. The electrodes are inserted through small burr holes in the skull, based on a plan created by a neurosurgeon from MR images. Once the electrodes have been placed at the targets, continuous high frequency pulses are delivered to achieve the desired effect.

The widespread adoption of DBS is due to its minimally-invasiveness, reversibility, and profound therapeutic effects. Recent work, however, has shown that inaccurate electrode placement can have significant adverse effects and reduce the therapeutic effect [2]. In particular, imperfect surgical planning due to difficulties in localizing anatomical and functional targets may be a contributing factor. Although electrophysiological exploration across several micro-electrodes near the planned target is possible, accurate electrode trajectory planning remains one of the most important phases of the surgical workflow.

Electrode trajectories are typically planned using co-registered T1-weighted and T2weighted pre-operative MR images. During planning, the target point is chosen in the registered images based on the particular disease to have the optimal therapeutic effect. Subsequently, the entry point is chosen such that the linear trajectory from the entry point to the target point poses the least risk to the patient.

Planning safe electrode trajectories requires a combined anatomical, procedural, and functional understanding of the situation. Junior neurosurgeons, who may not have achieved this understanding, are significantly more likely to encounter complications [3]. Thus, their training is essential. This requires two key components: a proficiency assessment method to monitor trainees' progress and a feedback mechanism to help trainees improve their understanding of the clinical situation and the appropriateness of their approach [4].

For this work, we proposed, implemented, and validated a training course for DBS planning. First, we developed and validated measures for objective assessment of planning proficiency. Next, we implemented objective feedback measures of the trajectory quality. Finally, we integrated the objective measures of proficiency with the feedback measures into a complete virtual training course for DBS planning.

\section{Previous Works}

In recent years, several software solutions have been introduced to facilitate DBS planning. Guo et al. introduced one of the first DBS planning platforms, which offered automated image registration, anatomical segmentation, and visualization for target localization [5]. Miocinovic et al. created the Cicerone system for DBS electrode placement, which also allows users to visualize the volume of tissue activated [6]. D'Haese et al. developed the CranialVault system for assistance through the pre-, intra-, and post-operative phases of the DBS workflow, including automatic prediction of the stimulation target point [7]. D'Albis et al. proposed the PyDBS system for DBS planning and post-operative assessment, including atlas-based segmentation, automatic stereotactic frame registration, and automatic segmentation of electrodes from post-operative CT images [8]. Commercial systems have been well-accepted in clinical practice [9], but tend to lack the features and flexibility offered by planning platforms designed for research.

In addition to software solutions for facilitating the DBS planning process, several algorithms have been proposed for automatically determining a suitable electrode trajectory for DBS. These methods typically rely on an anatomical segmentation of the brain and attempt to find the trajectory (or set of trajectories) which optimizes a cost function [10]-[15]. The cost function 
is usually based on expert consensus and penalizes trajectories which intersect or nearly intersect critical structures (e.g. sulci, ventricles). Additionally, Essert et al. have further attempted to adapt their cost function's parameters to match neurosurgeon preferences [16]. Moreover, "heat maps" based on the cost of every possible trajectory can be displayed to further assist operators [12], [13], [15], [17].

Alongside these studies, the development of neurosurgery simulators for training has also seen a recent increase in popularity, due to increasing fidelity and decreasing cost [18]. Training systems specifically targeting neurosurgery planning, however, remain few. Neubauer et al. introduced a simulator for endoscopic pituitary surgery which has visualization and navigation aids intended to help trainees better understand their plans [19]. Kockro et al. introduced the Dextrobeam, an extension on a neurosurgery planning system, which allows experts to guide novices through the anatomy and training process [20]. John et al. introduced and validated the VCath software for ventricular catheterization, which allows the user to practice planning the procedure from a tablet device [21].

To the best of our knowledge, no training tool specifically addressing DBS planning has been previously introduced.

\section{Methods}

In this section, we first introduce the measures for assessing proficiency in DBS trajectory planning developed in consultation with expert neurosurgeons. Next, we introduce the training course and its software implementation. Finally, we describe the study protocol used to validate the measures and the training approach.

\section{Primitive Metrics to Assess Trajectory Quality}

Angle from Reference Trajectory:

This metric measures the angle between the trajectory chosen by the operator and the reference trajectory defined by a DBS expert, which was used for patient treatment.

Distance from Reference Target Point:

This metric measures the distance between the target point chosen by the operator and the reference target point defined by a DBS expert, which was used for patient treatment.

\section{Trajectory Risk:}

This metric measures the risk value associated with the trajectory, based on segmentations of the target structure, ventricles, and sulci. The metric is computed using the aggregate cost function and weights proposed by Essert et al. [12]. The risk value is on the range $[0,1]$, where a lower score indicates a safer trajectory.

\section{Primitive Metrics to Assess Planning Efficiency and Completeness}

\section{Total Elapsed Time:}

This metric measures the total time it takes the user to plan the procedure, starting from when the user is first presented with the particular patient case and ending when the user has declared they are satisfied with the planned trajectory.

\section{Entry and Target Bounding Box:}

This metric measures how disparate the different trajectories the user tried were. This is computed from the volume of the bounding box containing all attempted entry and target points. 
Number of Slice Crosshair Movements:

This metric measures how many separate times the user views and interacts with the image slices. This is computed from the number of times the user moved the axial, coronal, and sagittal slice crosshair.

Path Length of Slice Intersection:

This metric measures how many different image slices in each of the axial, coronal, and sagittal planes the user viewed. This provides a sense of how much the operator inspected the images during the planning.

Verification of Trajectory:

This metric measures how well the operator has verified that the trajectory does not intersect any of the anatomy using the patient images. It is calculated as the proportion of the trajectory the operator has viewed in each of the axial, coronal, and sagittal slices.

Verification of Registration:

Because certain anatomies are visible in the T1 MRI image and others in the T2 MRI image, the two imaging modalities must be registered. This metric computes how much the user has checked that the images are registered and the anatomical segmentation is acceptable, based on the extent to which the user has viewed the overlaid images.

\section{Overall Measure of Proficiency}

Each of the primitive metric assesses the operator's proficiency in a particular aspect of the procedure. We propose that a combined measure of proficiency, based on the primitive metrics, will have more discriminatory power to distinguish operators of different skill levels.

To this end, we implemented a non-parametric extension of the z-score normalization method [22]. Due to the skewed distribution of primitive metrics, we used a weighted sum of percentile ranks relative to the expert population, where the weights necessarily sum to one. For this application, equal weights were used. In practice, the combined measure of proficiency $c_{i}$ for the $i$ th trainee can be written as follows (where $r_{i, j}$ is their percentile rank amongst the expert values for the $j$ th primitive metric using the average rank in case of ties and $N_{M}$ is the number of primitive metrics).

$$
c_{i}=\frac{1}{N_{M}} \sum_{j=1}^{N_{M}} r_{i, j}
$$

Equation 1

\section{Feedback Components}

To assist trainees in understanding the clinical situations, we propose providing feedback during training. Based on expert consultation, trainees were provided with the measures of trajectory quality as feedback during planning. These measures were provided both visually, to provide an intuitive sense of the trajectory quality, and numerically, to provide precise information (Error! Reference source not found.).

Distance to Reference Target Point:

This metric was displayed visually through the color of the target point.

Angle from Reference Trajectory:

This metric was displayed visually through the color of the entry point.

\section{Trajectory Risk:}

This metric was displayed visually through the color of the trajectory line from the entry point to the target point. 


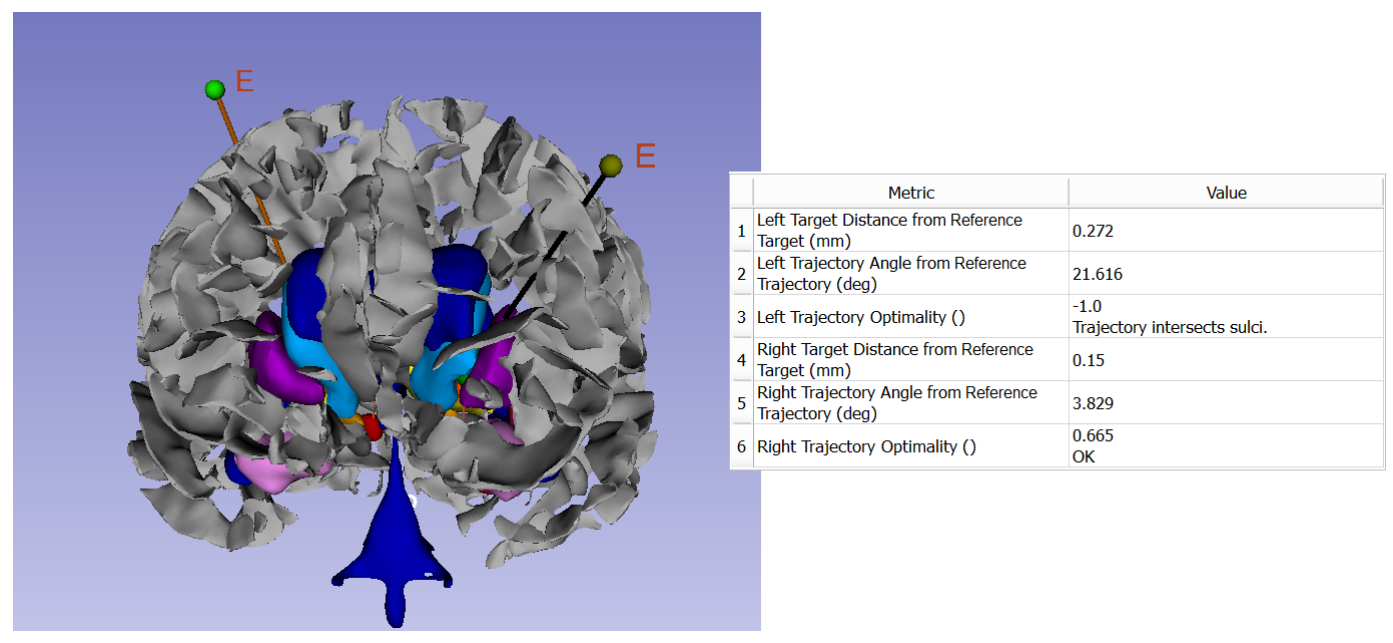

Figure 1: Illustration of visual (left) and numeric (right) feedback provided to trainees as they plan their electrode trajectories.

For each feedback component, a high-quality trajectory was indicated in green and a lowquality trajectory was indicated in red. For the visual feedback on Trajectory Risk, unacceptable trajectories (missing the target anatomy or intersecting critical anatomy) were displayed in black.

\section{Training Course}

Based on expert consultation, we propose a training course involving five phases. During each phase, the trainee completes two DBS plans: one targeting the subthalamic nucleus (STN) and one targeting the internal part of the globus pallidus (GPI). With the exception of the first phase, the training course is designed to be completed by each trainee on their own, without any instruction from a DBS expert.

\section{Phase 1: Introduction:}

The trainee is exposed to the entire DBS planning process and all feedback components. This phase allows the trainee to familiarize themselves with the planning software; the trainee may ask questions about how to use the software.

Phase 2: Pre-Training Assessment:

The trainee must go through the complete DBS planning process, where no feedback is provided. This phase assesses the trainee's DBS planning proficiency at the beginning of the training course.

\section{Phase 3: Training Phase A:}

The trainee is provided with a pre-planned target point. Thus, the trainee plans only the entry point of the trajectory, with feedback provided throughout. This phase helps the trainee learn how to plan the entry point based on the cranial anatomy, given the target point has been appropriately identified.

\section{Phase 4: Training Phase B:}

The trainee must go through the complete DBS planning process, with feedback provided throughout. This phase lets the trainee practice the entire planning process, learning from the feedback provided on their trajectory quality. 


\section{Phase 5: Post-Training Assessment:}

The trainee must again go through the complete DBS planning process, where no feedback is provided. This phase assesses the trainee's DBS planning proficiency at the end of the training course.

Throughout, all measures of proficiency are recorded in the background. The measures of proficiency during the pre-training and post-training assessment phases can be compared to assess the trainee's improvement throughout the training course.

\section{Software Implementation}

The training system for DBS planning was implemented in the 3D Slicer (www.slicer.org) environment (Error! Reference source not found.) and may be installed on any Linux, Mac, or Windows computer. The DBS planning interface was based on the PyDBS software, which was used to register and segment the MRI images and allowed the patient cases to be readily loaded, visualized, and planned [8]. The Perk Tutor platform (www.perktutor.org) was used to capture the planning process, calculate all measures of proficiency, and compute the feedback components [23].

\section{Study Protocol}

To validate whether the proposed training course has added value in the training process, we carried out a validation study. For this study, two expert neurosurgeons and six neurosurgery trainees were recruited at three centers in France and one center in Canada. Neurosurgeons who had planned at least 100 total DBS were considered experts; others were considered novices.

Prior to beginning the training course, participants completed a questionnaire about their previous experience in DBS, were shown a brief demonstration on how to use the software, and

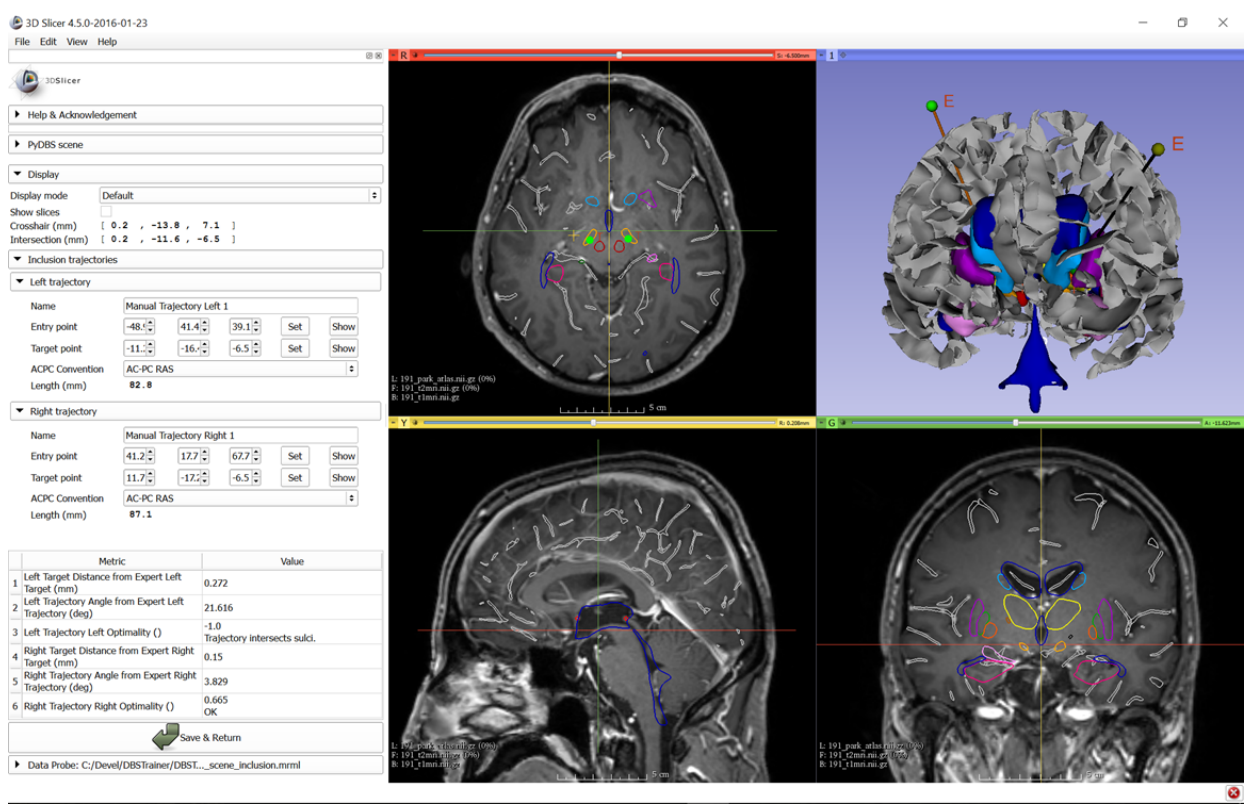

Figure 2: Screenshot of the simplified PyDBS interface with visual and numeric feedback used for training for DBS planning. 
allowed to familiarize themselves with the software. Subsequently, each participant followed the training course for DBS planning (expert participants skipped the two training phases). The ten patient cases were selected from real, anonymized patient data from patients with Parkinson's disease, representing a variety of anatomical variations. Participants planned the trajectories at the inclusion phase of the workflow: patients had already been identified as good candidates for DBS, the target anatomy had been previously identified (STN or GPI), the T1-weighted and T2weighted MR images had already been taken, and the T2-weighted MR image along with an atlasbased segmentation had already been registered to the T1-weighted MR image using PyDBS.

At the end of the training course, participants rated how difficult they thought each case was. Participants also completed a survey to assess the acceptance of the training software, based on the previously validated UTAUT [24]. The survey included items concerning the usefulness of the provided feedback, the usefulness of the training course, and participants' interaction with the software.

\section{Statistical Analysis}

All measures of proficiency were tested for evidence of construct validity (i.e. their ability to discriminate novices from experts at baseline) by Wilcoxon rank-sum test ( $\alpha=0.05)$. Spearman's rank correlation test was used to determine the correlation between each proficiency measure and the number of DBS previously planned $(\alpha=0.05)$. The added value of the training course for DBS planning (i.e. novices' improvement in proficiency measures following training) was assessed by Wilcoxon signed-rank test $(\alpha=0.05)$. In all cases, Cliff's $\Delta$ was used to measure effect size.

\section{Results}

\section{Construct Validity of Proficiency Measures}

The expert group had more initial median experience planning DBS (134.5 vs. 0.0 ), as well as more experience observing, assisting, and performing DBS than the novice group (Table 1). Furthermore, experts indicated feeling more comfortable planning DBS.

Table 1: Demographic information for the novice and expert groups.

\begin{tabular}{lll}
\hline Demographic & Novice & Expert \\
\hline Gender (M/F) & $5 / 1$ & $1 / 1$ \\
Age & $32.5(27.0-32.5)$ & $37.0(35.0-39.0)$ \\
Residency Program (yrs) & $5.3(5.0-5.5)$ & $5.0(5.0-5.0)$ \\
Residency Completed (yrs) & $4.0(2.5-5.5)$ & $5.0(5.0-5.0)$ \\
Years in Practice & $2.0(0.0-5.0)$ & $8.0(5.0-11.0)$ \\
DBS Observed & $20.0(5.0-100.0)$ & $1058.0(100.0-2016.0)$ \\
DBS Planned & $0.0(0.0-45.0)$ & $134.5(100.0-169.0)$ \\
DBS Performed & $0.0(0.0-3.0)$ & $99.5(30.0-169.0)$ \\
DBS Assisted & $20.0(5.0-100.0)$ & $182.5(150.0-215.0)$ \\
Planning Comfort & $1.0(0.0-5.0)$ & $8.0(8.0-8.0)$ \\
\hline
\end{tabular}

Values indicated are medians (inter-quartile range). 
Table 2: Primitive metrics for the novice and expert groups.

\begin{tabular}{lllll}
\hline Metric & Novice & Expert & Effect size $(p$ value $)$ & Correlation $(p$ value $)$ \\
\hline Angle from reference trajectory $\left({ }^{\circ}\right)$ & $15.44(3.95-20.79)$ & $7.45(6.33-13.54)$ & $-0.26(0.29)$ & $-0.44(0.01)^{*}$ \\
Distance from reference target $(\mathrm{mm})$ & $3.91(2.97-6.23)$ & $3.02(2.11-6.07)$ & $-0.21(0.37)$ & $-0.08(0.66)$ \\
Trajectory risk (0-1) & $0.66(0.62-0.68)$ & $0.64(0.62-0.67)$ & $-0.09(0.73)$ & $-0.10(0.57)$ \\
Total elapsed time (s) & $182.05(125.98-373.40)$ & $165.64(153.12-174.68)$ & $-0.25(0.52)$ & $0.06(0.81)$ \\
Number of entry movements & $2.50(1.00-6.00)$ & $1.50(1.00-3.00)$ & $-0.15(0.55)$ & $-0.45(0.01)^{*}$ \\
Number of target movements & $0.00(0.00-0.50)$ & $3.00(0.00-7.00)$ & $0.38(0.06)$ & $0.34(0.06)$ \\
Entry bounding box $\left(\mathrm{mm}^{3}\right)$ & $1095.58(27.83-12871.24)$ & $0.72(0.00-1300.06)$ & $-0.39(0.11)$ & $-0.52(<0.01)^{*}$ \\
Target bounding box $\left(\mathrm{mm}^{3}\right)$ & $0.00(0.00-0.00)$ & $0.01(0.00-0.31)$ & $0.33(0.06)$ & $0.28(0.12)$ \\
Number of slice crosshair movements & $69.00(39.00-100.00)$ & $32.00(27.50-42.00)$ & $-0.79(0.02)^{*}$ & $-0.32(0.23)$ \\
Path length of slice intersection (mm) & $1600.00(1137.29-1984.41)$ & $1651.62(1438.24-2211.80)$ & $0.13(0.77)$ & $-0.03(0.91)$ \\
Verification of trajectory-axial $(\%)$ & $8.07(0.00-46.87)$ & $10.64(3.22-57.50)$ & $0.18(0.46)$ & $0.03(0.86)$ \\
Verification of trajectory-coronal $(\%)$ & $9.26(0.00-30.61)$ & $72.11(34.08-100.00)$ & $0.56(0.02)^{*}$ & $0.34(0.06)$ \\
Verification of trajectory-sagittal $(\%)$ & $0.00(0.00-17.78)$ & $4.55(0.00-16.90)$ & $0.13(0.58)$ & $0.18(0.34)$ \\
Verification of registration $(\%)$ & $0.00(0.00-0.00)$ & $0.00(0.00-0.00)$ & $0.00(1.00)$ & N/A (N/A) \\
\hline Values are medians (inter-quartin
\end{tabular}

Values are medians (inter-quartile range). Effect size is computed using Cliff's $\Delta$ (significance level of rank-sum test). Correlation is computed using Spearman's $\rho$ (significance level of correlation). Asterisk indicates a significant difference or significant correlation.
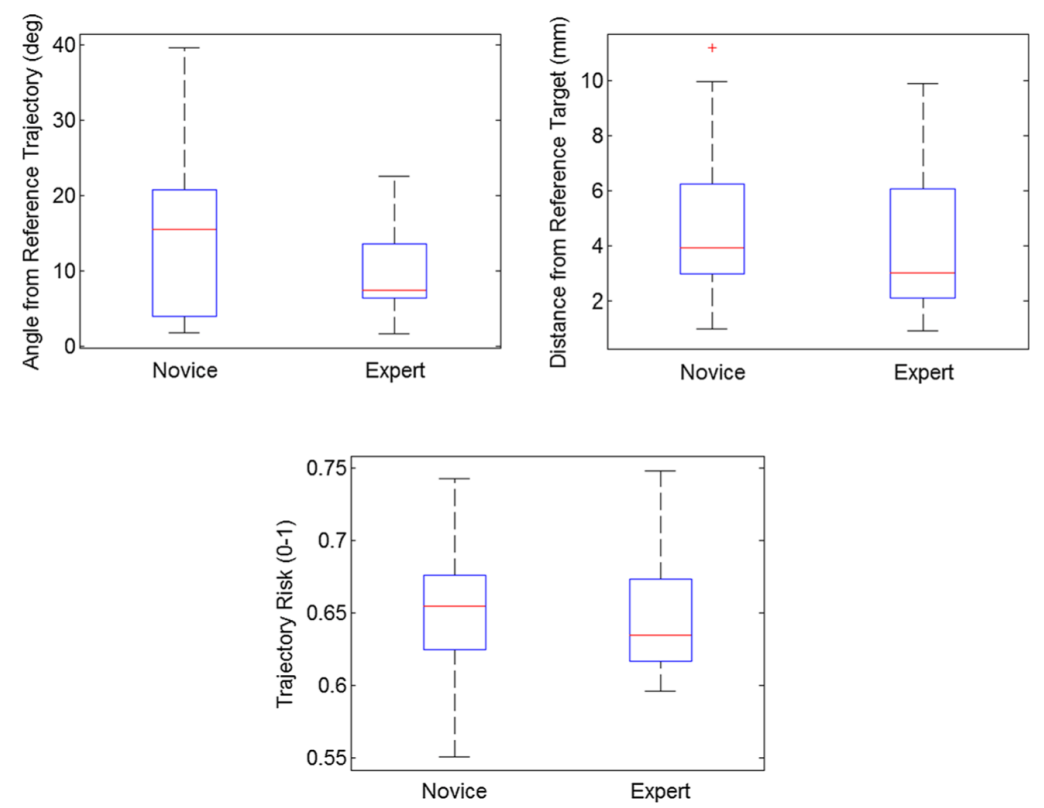

Figure 3: Overall measure of proficiency for novices versus experts (left) and correlation with the number of DBS previously planned (right).

In total, novices and experts were assessed via fourteen primitive metrics of proficiency and the overall measure of proficiency. Prior to training, two primitive metrics had a significant difference in operator performance between novices and experts. Furthermore, three primitive metrics showed a significant correlation with the number of DBS previously planned (Table 2). Experts performed the procedures with better trajectory quality metrics than the novices, but these differences were not significant (Fig. 3). 

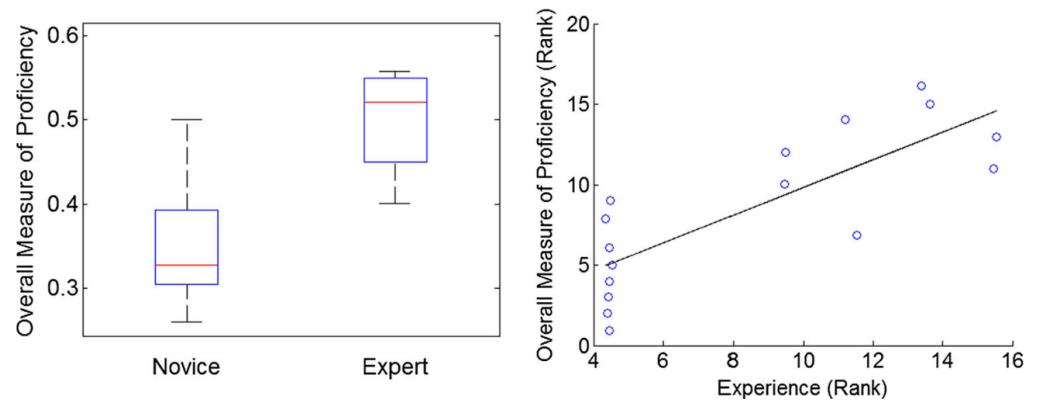

Figure 4: Overall measure of proficiency for novices versus experts (left) and correlation with the number of DBS previously planned (right).

The overall measure of proficiency exhibited both a significant difference between the novice and expert groups $(p=0.008, \Delta=0.88)$, and it correlated significantly with number of DBS previously planned $(p<0.001, \rho=0.81 ;$ Fig.4).

\section{Added Value of Training Course}

After training, novices were again assessed using the fourteen primitive metrics and the overall measure of proficiency. Due to time constraints, one novice was only able to complete the pre-training phase, and his results were not included for pre-training vs. post-training analysis. Two primitive metrics showed a statistically significant improvement from pre-training to post-training (Table 3). All measures of trajectory quality improved, but this improvement was significant for only the distance from the reference target (Fig.5).

The overall proficiency measure showed a statistically significant improvement from pretraining to post-training $(p=0.002, \Delta=1.00$; Fig.6), with every novice showing improvement. Furthermore, novices were more confident in planning DBS after completing the training course

Table 3: Primitive metrics for the novice groups before and after training.

\begin{tabular}{llll}
\hline Metric & Pre-training & Post-training & Effect size $(p$ value $)$ \\
\hline Angle from reference trajectory $\left({ }^{\circ}\right)$ & $16.41(7.70-21.25)$ & $11.13(6.44-17.09)$ & $-0.20(0.09)$ \\
Distance from reference target $(\mathrm{mm})$ & $3.90(2.81-6.23)$ & $1.86(1.10-4.10)$ & $-0.60(0.01)^{*}$ \\
Trajectory risk $(0-1)$ & $0.66(0.62-0.67)$ & $0.64(0.62-0.70)$ & $0.00(0.52)$ \\
Total elapsed time $(\mathrm{s})$ & $170.34(118.61-221.40)$ & $141.33(92.64-194.81)$ & $-0.40(0.22)$ \\
Number of entry movements & $3.00(2.00-7.50)$ & $4.001 .50-5.50)$ & $-0.35(0.15)$ \\
Number of target movements & $0.00(0.00-1.00)$ & $0.00(0.00-1.50)$ & $-0.50(0.61)$ \\
Entry bounding box $\left(\mathrm{mm}^{3}\right)$ & $3335.24(135.50-19639.20)$ & $1679.51(74.22-6861.38)$ & $-0.30(0.03) *$ \\
Target bounding box $\left(\mathrm{mm}^{3}\right)$ & $0.00(0.00-0.00)$ & $0.00(0.00-0.00)$ & $-0.70(0.34)$ \\
Number of slice crosshair movements & $55.50(38.00-77.00)$ & $44.40(37.00-51.00)$ & $-0.20(0.16)$ \\
Path length of slice intersection $(\mathrm{mm})$ & $1600.00(979.50-1931.47)$ & $1711.90(887.01-2654.31)$ & $-0.20(0.31)$ \\
Verification of trajectory-axial $(\%)$ & $13.42(0.00-46.87)$ & $5.05(2.00-97.18)$ & $-0.10(0.58)$ \\
Verification of trajectory-coronal $(\%)$ & $9.26(0.00-19.77)$ & $9.90(0.00-28.71)$ & $0.00(0.64)$ \\
Verification of trajectory-sagittal $(\%)$ & $0.00(0.00-5.98)$ & $3.47(0.00-17.69)$ & $-0.30(0.68)$ \\
Verification of registration $(\%)$ & $0.00(0.00-0.00)$ & $0.00(0.00-0.00)$ & $0.00(1.00)$ \\
\hline
\end{tabular}

Values are medians (inter-quartile range). Effect size is computed using Cliff's $\Delta$ (significance level of the rank-sum test). Asterisk indicates a significant difference. 

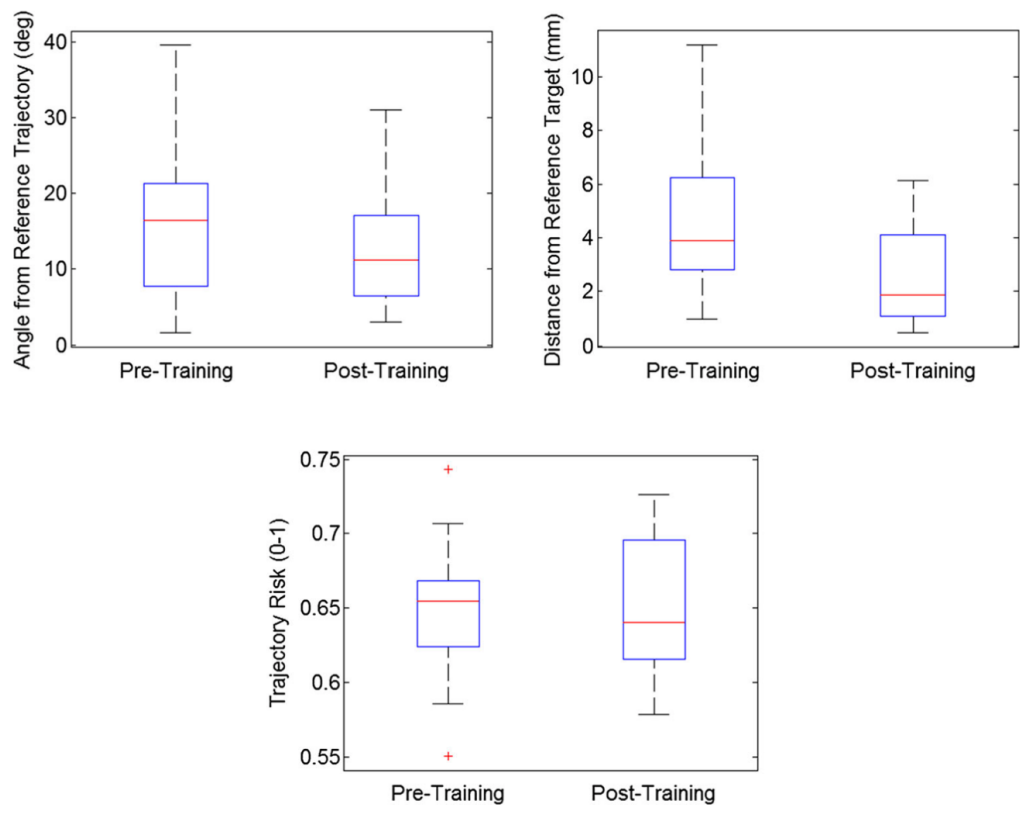

Figure 5: Plots of pre-training versus post-training trajectory quality metrics: angle from reference trajectory (top-left), distance from reference trajectory (top-right), and trajectory risk (middle).

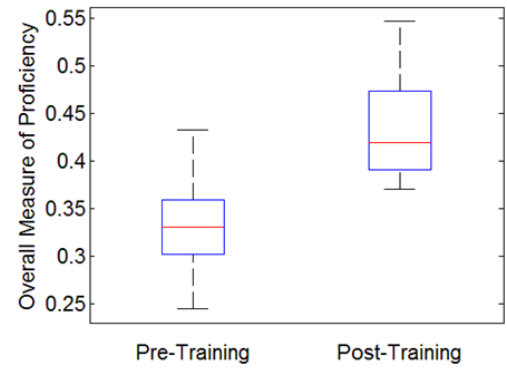

Figure 6: Overall measure of proficiency pre-training versus post-training. Higher values indicate greater similarity to experts.

and had less perceived difficulty with the post-training cases, but these differences were not significant ( $p=0.13$ and $p=0.18$, respectively).

In all cases, novices' post-training primitive metrics did not exceed experts' primitive metrics. The overall proficiency measure was smaller for novices post-training than for experts with medium effect size, but the difference was not significant ( $p=0.19, \Delta=0.50)$.

\section{Qualitative Evaluation of Training Software}

On average, novice participants agreed that training for DBS planning with the software was a good idea ( 6.6 out of 7 ), the feedback provided by the system improved their understanding of the patient cases (6.4 out of 7), and the software would be a useful part of a training curriculum 


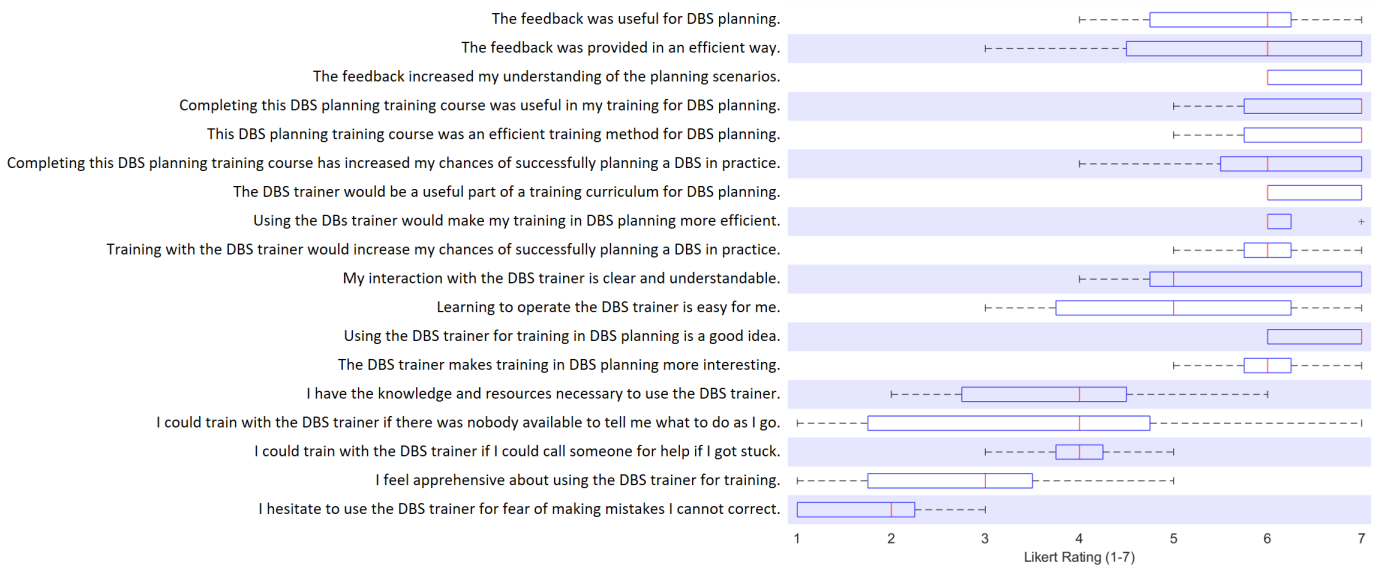

Figure 7: Survey responses for novices after completing the training course using a 7-point Likert scale (1 indicates strong disagreement, 7 indicates strong agreement).

(6.4 out of 7). Although novices were receptive to the training software, they found it difficult to operate without assistance (3.6 out of 7). Full survey results are shown in Fig 7.

We installed the training software on a variety of different user-grade Linux, Mac, and Windows computers. In all cases, installation took less than thirty minutes, and performance was deemed acceptable based on informal operator feedback about responsiveness and image rendering quality.

\section{Discussion}

While most primitive metrics showed no significant difference between the novice and expert groups, the overall measure of proficiency demonstrated construct validity. Thus, it can be used as a scale for trainee assessment throughout a training course, based on a small set of expert benchmarks. Between pre-training and post-training, all novices improved with respect to this measure. This indicates that the proposed training course has added value in the learning process for DBS planning.

One of the main limitations of this study is sample size. DBS is not performed regularly at many centers; thus, the expert population is limited. Results indicate a sample size of 40 ( $\alpha=0.05$; $\beta=0.80$ ) would be required to determine validity. Furthermore, the measures of proficiency should be validated against a gold-standard measure of proficiency (e.g. checklist or global rating scale). To the best of our knowledge, no gold-standard exists for DBS planning. For this study, we validated the proposed measures of proficiency against DBS planning experience. Unfortunately, this does not account for instances where experts make mistakes or novices benefit from a stroke of fortune. We suggest this partially accounts for the overlap between the novice and expert groups with respect to the measures of proficiency.

Because the training setup is exclusively software-based, it can be run on any modern computer. Thus, it could be readily installed in neurosurgery centers and allow trainees to independently practice and self-assess on a dedicated training station. Because the training setup is based on PyDBS, whenever PyDBS is used in practice to confirm DBS plans (as it is at the Centre Hospitalier Universitaire de Rennes), real patient cases can be readily transferred, in accordance 
with ethical bodies, to the training system. This will allow trainees to practice on recent, clinically relevant cases with results that translate directly to clinical proficiency. Moreover, as the setup is based on Perk Tutor, new measures of proficiency can be added by expert teachers to assess aspects of the procedure that are specific to their patient cases or to emphasize specific skills for their trainees to work on. In addition, the weighting scheme for the overall measure of proficiency could be adjusted to have optimal discriminatory power, based on expert consensus, or on a caseby-case basis to emphasize particular skills.

Two primitive metrics depend on a reference trajectory. For our validation study, the reference trajectory was chosen by an expert neurosurgeon to treat the patient in practice. In general, it could be computed automatically or taken as an aggregate of several expert trajectories.

This training course does not represent a complete DBS training curriculum, but rather, a self-guided component which trainees can complete on their own. We envision that this training course could be extended with more patient cases representing different targets and diseases, and it could be customized to teach particular aspects of DBS planning. The proposed training course would allow trainees to learn and practice the basics of DBS planning, and to monitor their own progress prior to planning DBS under expert supervision: an early-stage component of a larger DBS training curriculum.

\section{Conclusion}

We have developed, implemented, and validated a training course for DBS planning. Objective measures of proficiency were developed and validated to quantitatively assess and monitor proficiency in DBS planning, including an overall measure of proficiency which assesses performance across many different components of the planning process. Subsequently, a training course was implemented and completed by a group of trainees. The training course's efficacy was validated by monitoring the improvement in trainees' measures of proficiency. The training course fits into the context of the worldwide shift to a competency-based model of medical education.

\section{Acknowledgements}

Matthew S. Holden was supported by the Natural Sciences and Engineering Research Council (NSERC) Canada Graduate Scholarship. Travel was supported by the Mitacs Globalink Research Award - Campus France and the Rennes Metropole Mobility Grant. Gabor Fichtinger was supported as a Cancer Care Ontario Research Chair in Cancer Imaging. This work was financially supported as a Collaborative Health Research Project (CHRP \#127797), a joint initiative between the Natural Sciences and Engineering Research Council of Canada (NSERC) and the Canadian Institutes of Health Research (CIHR).

\section{Compliance with Ethical Standards}

Conflict of Interest

All authors declare that they have no conflict of interest.

\section{Ethical Approval}

All procedures performed in studies involving human participants were in accordance with the ethical standards of the institutional and/or national research committee and with the 1964 Helsinki Declaration and its later amendments or comparable ethical standards. 
Informed Consent

All participation was voluntary. Written informed consent was obtained from all participants in this study.

\section{References}

[1] P. Krack, M. I. Hariz, C. Baunez, J. Guridi, and J. A. Obeso, "Deep brain stimulation: From neurology to psychiatry?," Trends in Neurosciences, vol. 33, no. 10. pp. 474-484, 2010.

[2] M. K. York, E. A. Wilde, R. Simpson, and J. Jankovic, "Relationship between neuropsychological outcome and DBS surgical trajectory and electrode location," J. Neurol. Sci., vol. 287, no. 1-2, pp. 159-171, 2009.

[3] J. Voges, Y. Waerzeggers, M. Maarouf, R. Lehrke, A. Koulousakis, D. Lenartz, and V. Sturm, "Deep-brain stimulation: long-term analysis of complications caused by hardware and surgery--experiences from a single centre.," J. Neurol. Neurosurg. Psychiatry, vol. 77, no. 7, pp. 868-72, 2006.

[4] D. A. Rogers, G. Regehr, T. R. Howdieshell, K. A. Yeh, and E. Palm, "The impact of external feedback on computer-assisted learning for surgical technical skill training," Am. J. Surg., vol. 179, no. 4, pp. 341-343, Apr. 2000.

[5] T. Guo, K. W. Finnis, A. G. Parrent, and T. M. Peters, "Visualization and navigation system development and application for stereotactic deep-brain neurosurgeries," Comput. Aided Surg., vol. 11, no. 5, pp. 231-239, 2006.

[6] S. Miocinovic, A. M. Noecker, C. B. Maks, C. R. Butson, and C. C. Mclntyre, "Operative Neuromodulation: Volume 2: Neural Networks Surgery," D. E. Sakas and B. A. Simpson, Eds. Vienna: Springer Vienna, 2007, pp. 561-567.

[7] P.-F. D`Haese, S. Pallavaram, R. Li, M. S. Remple, C. Kao, J. S. Neimat, P. E. Konrad, and B. M. Dawant, "CranialVault and its CRAVE tools: A clinical computer assistance system for deep brain stimulation (DBS) therapy," Med. Image Anal., vol. 16, no. 3, pp. 744-753, 2012.

[8] T. D`Albis, C. Haegelen, C. Essert, S. Fernandez-Vidal, F. Lalys, and P. Jannin, "PyDBS: an automated image processing workflow for deep brain stimulation surgery," Int. J. Comput. Assist. Radiol. Surg., vol. 10, no. 2, pp. 117-128, 2014.

[9] G. G. Heuer, K. A. Zaghloul, J. L. Jaggi, and G. H. Baltuch, "Use of an integrated platform system in the placement of deep brain stimulators," Neurosurgery, vol. 62, no. 3 Suppl 1, pp. 245-247, Mar. 2008.

[10] E. J. L. Brunenberg, A. Vilanova, V. Visser-Vandewalle, Y. Temel, L. Ackermans, B. Platel, and B. M. ter Haar Romeny, "Automatic Trajectory Planning for Deep Brain Stimulation: A Feasibility Study," in Medical Image Computing and Computer-Assisted Intervention -MICCAI 2007: 10th International Conference, Brisbane, Australia, October 29 - November 2, 2007, Proceedings, Part I, N. Ayache, S. Ourselin, and A. Maeder, Eds. Berlin, Heidelberg: Springer Berlin Heidelberg, 2007, pp. 584-592.

[11] P. Tirelli, E. De Momi, N. A. Borghese, and G. Ferrigno, "An intelligent atlas-based planning system for keyhole neurosurgery," Int. J. Comput. Assist. Radiol. Surg., vol. 4, no. 1, pp. 8586, 2009.

[12] C. Essert, C. Haegelen, F. Lalys, A. Abadie, and P. Jannin, "Automatic computation of electrode trajectories for Deep Brain Stimulation: a hybrid symbolic and numerical approach," Int. J. Comput. Assist. Radiol. Surg., vol. 7, no. 4, pp. 517-532, 2011.

[13] S. Beriault, F. Al Subaie, D. L. Collins, A. F. Sadikot, and G. B. Pike, "A multi-modal approach to computer-assisted deep brain stimulation trajectory planning," Int. J. Comput. Assist. 
Radiol. Surg., vol. 7, no. 5, pp. 687-704, 2012.

[14] R. R. Shamir, L. Joskowicz, I. Tamir, E. Dabool, L. Pertman, A. Ben-Ami, and Y. Shoshan, "Reduced risk trajectory planning in image-guided keyhole neurosurgery," Med. Phys., vol. 39, no. 5, pp. 2885-2895, 2012.

[15] Y. Liu, P. E. Konrad, J. S. Neimat, S. B. Tatter, H. Yu, R. D. Datteri, B. A. Landman, J. H. Noble, S. Pallavaram, B. M. Dawant, and P.-F. D'Haese, "Multisurgeon, Multisite Validation of a Trajectory Planning Algorithm for Deep Brain Stimulation Procedures," Biomed. Eng. IEEE Trans., vol. 61, no. 9, pp. 2479-2487, Sep. 2014.

[16] C. Essert, S. Fernandez-Vidal, A. Capobianco, C. Haegelen, C. Karachi, E. Bardinet, M. Marchal, and P. Jannin, "Statistical study of parameters for deep brain stimulation automatic preoperative planning of electrodes trajectories," Int. J. Comput. Assist. Radiol. Surg., vol. 10, no. 12, pp. 1973-1983, 2015.

[17] N. V Navkar, N. V Tsekos, J. R. Stafford, J. S. Weinberg, and Z. Deng, "Visualization and Planning of Neurosurgical Interventions with Straight Access," in Information Processing in Computer-Assisted Interventions: First International Conference, IPCAI 2010, Geneva, Switzerland, June 23, 2010. Proceedings, N. Navab and P. Jannin, Eds. Berlin, Heidelberg: Springer Berlin Heidelberg, 2010, pp. 1-11.

[18] A. Alaraj, M. G. Lemole, J. H. Finkle, R. Yudkowsky, A. Wallace, C. Luciano, P. P. Banerjee, S. H. Rizzi, and F. T. Charbel, "Virtual reality training in neurosurgery: Review of current status and future applications," Surg Neurol Int, vol. 2, p. 52, 2011.

[19] A. Neubauer, S. Wolfsberger, M. T. Forster, L. Mroz, R. Wegenkittl, and K. Buhler, "Advanced virtual endoscopic pituitary surgery," IEEE Trans Vis Comput Graph, vol. 11, no. 5, pp. 497-507, 2005.

[20] R. A. Kockro, A. Stadie, E. Schwandt, R. Reisch, C. Charalampaki, I. Ng, T. T. Yeo, P. Hwang, L. Serra, and A. Perneczky, "A collaborative virtual reality environment for neurosurgical planning and training," Neurosurgery, vol. 61, no. 5 Suppl 2, pp. 379-391, Nov. 2007.

[21] N. W. John, N. I. Phillips, L. a. Cenydd, D. Coope, N. Carleton-Bland, I. Kamaly-Asl, and W. P. Gray, "A Tablet-Based Virtual Environment for Neurosurgery Training," Presence, vol. 24, no. 2, pp. 155-162, May 2015.

[22] B. Allen, V. Nistor, E. Dutson, G. Carman, C. Lewis, and P. Faloutsos, "Support vector machines improve the accuracy of evaluation for the performance of laparoscopic training tasks," Surg Endosc, vol. 24, no. 1, pp. 170-178, Jan. 2010.

[23] T. Ungi, D. Sargent, E. Moult, A. Lasso, C. Pinter, R. C. McGraw, and G. Fichtinger, "Perk Tutor: An Open-Source Training Platform for Ultrasound-Guided Needle Insertions," IEEE Trans. Biomed. Eng., vol. 59, no. 12, pp. 3475-3481, Dec. 2012.

[24] V. Venkatesh, M. G. Morris, G. B. Davis, and F. D. Davis, "User Acceptance of Information Technology: Toward a Unified View," MIS Q., vol. 27, no. 3, pp. 425-478, 2003. 